\title{
Flannery 0'Connor: la violencia y la gracia
}

\section{Marina FE \\ Universidad Nacional Autónoma de México}

\begin{abstract}
Este artículo es resultado de la lectura de algunos relatos de Flannery O'Connor desde la perspectiva del llamado "gótico americano". El propósito es explorar el tema de la violencia que predomina en la narrativa de esta autora acompañado de su idea de la gracia, en el sentido cristiano del término. O'Connor era una escritora profundamente católica y en sus cuentos busca que la gracia llegue no sólo a sus personajes sino también a sus lectores. Se trata de una suerte de revelación que puede tener lugar en su conciencia a partir de una experiencia terrible que los transforma.
\end{abstract}

PALABRAS CLAVE: gótico, violencia, gracia, revelación.

In this article I read some of Flannery O'Connor's short stories from the perspective of the so-called "American Gothic". My purpose is to explore the subject of violence which is central in the narrative of this author together with her idea of grace, in the Christian sense of the term. O'Connor was a profoundly catholic writer and in her stories she expects that grace will touch not only her characters but also her readers. It is a sort of revelation that can take place in the conscience as the result of a terrible experience that transforms them.

KEY WORDS: gothic, violence, grace, revelation.

Acosada desde muy joven por el lobo que mataría a su padre, el lupus, Flannery O'Connor estaba obsesionada por la enfermedad y la muerte. En su colección de cuentos Everything That Rises Must Converge explora estos estados del cuerpo que tarde o temprano todos debemos enfrentar, así como diferentes formas de violencia que algunos de sus personajes deben sufrir para tener una suerte de revelación, algo así como las epifanías de Joyce, o los "moments of being", a la manera de Woolf, aunque ella se refiere a éstos como "moments of grace".

O’Connor se inscribe dentro de lo que se conoce como el gótico americano, y más específicamente, el gótico sureño. Es ésta una narrativa de la violencia, de una sociedad enferma con familias disfuncionales donde los individuos viven en una peculiar soledad, amenazados por el mundo exterior pero también por su propia realidad interior, eso que para Bajtin sería un "grotesco de cámara, una especie de carnaval que el individuo representa en soledad, con la conciencia agudizada de su aislamiento" (40) y 
donde se descubren solos, frente a una exterioridad amenazante y sombría. Se trata, en este caso, de personas (y personajes) que rompen con las normas de su comunidad:

The Southern experience was (is) still very much an affair of the complex patterns of community life, with the comings and goings of individuals taking place within a clearly recognized set of expectations and assumptions. In that kind of established social context, individual behaviour ran along expected forms, so there were certain agreed upon limits and standards of human conduct. Anything truly deviant, genuinely aberrant, could therefore stand out, since there was something against which it could be measured and identified (Rubin: 143).

Más que una crítica a la sociedad, O'Connor, lo mismo que Faulkner antes que ella, trata de mostrar el lado oscuro de la vida en el sur de Estados Unidos y de los individuos inmersos en una realidad que no acaban de comprender y con la que no saben cómo lidiar. Después de la derrota en la guerra civil y a causa del sentimiento de humillación que trajo consigo, el problema fundacional de esta cultura protestante, el debate entre el bien y el mal, produciría la obsesión con el pecado como origen de todo. Aquí el individuo, máximo baluarte de esta sociedad, está solo y su crisis es personal y privada. El gótico sureño es quizá la quintaesencia del llamado "gótico americano", y en la narrativa de O'Connor descubrimos "the secret center of the Gothic structure, where boundaries break down, where life and death become confused, where images of birth and sexuality proliferate in complex displacements" (Kahane: 338).

Los personajes de Faulkner viven encerrados en sí mismos, en su desolación y en soledad, en su locura y su pasión. Y también los personajes de O'Connor están solos, a pesar de que viven dentro de una comunidad en condiciones aparentemente normales. Sin embargo, ya no se trata aquí del mismo sur de Faulkner, sino más bien: "The grotesqueness of much of the death-dealing satire of the author's own ambivalent fascination with the great crisis that disrupted the South in the late fifties and early sixties: the rise of the civil rights movement" (Di Renzo: 197).

Flannery O'Connor, una escritora profundamente católica en el sur protestante de Estados Unidos, presenta en sus cuentos y novelas, llenos de humor e ironía, personajes blancos obsesionados por visiones del infierno y la salvación, quienes tienen que confrontar el mal para descubrir el lado oscuro de su naturaleza absolutamente humana e imperfecta. Ella pretendía que sus relatos fueran entendidos como parábolas que mostraran al lector laico la manifestación de la gracia, y con este propósito, de acuerdo con Samuel Chase y la misma autora:

She had to flatten her fictional world, to distort it cruelly in order to make room for the Christian mystery that was to arise from and within it: "It should reinforce our sense of the supernatural by grounding concrete, observable reality. And in such a world, in such a palpable physical and visible prison, violence is strangely capable of returning my characters to reality and preparing them to accept their moment of grace" (Chase: 89-90). 
Así, enfrentados a situaciones de violencia y a partir de estos momentos de crisis e incluso de espanto, es como sus personajes podrán acceder a la gracia, a su peculiar e individual manifestación.

La gracia es un don que Dios concede para que el alma se transforme y se eleve hasta participar de la naturaleza divina, y gracias a ella se puede descubrir, a partir del enfrentamiento con otro orden de realidad y de conciencia, una verdad, una revelación a la que no se habría podido llegar de ninguna otra manera. En su narrativa, es la violencia a la que se someten sus personajes lo que, paradojicamente, les permite el acceso a la gracia. En su libro Mystery and Manners dice la autora: "I have found that violence is strangely capable of returning my characters to reality and preparing them to accept their moment of grace" (144).

Pero no sólo los lectores pueden no llegar a reconocer o entender estos momentos, tampoco se puede saber realmente si los mismos personajes son capaces de acceder a la gracia y aprovechar esta oportunidad de transformación y de cambio, o dejarla pasar y seguir siendo los mismos de siempre. O'Connor no lo dice, no pretende darle una lección a nadie, y es muy probable que si no estuviéramos enterados como lectores de sus creencias católicas, al terminar de leer sus cuentos no pensaríamos nunca que podría haber, como en las parábolas, otra lectura, y que hay que descubrir, como si se tratara de un misterio, en qué consiste para cada personaje su momento de gracia.

En su colección de cuentos Everything That Rises Must Converge, Flannery O'Connor explora la posibilidad de leer y entender de manera diferente. Lectora del teólogo jesuita Pierre Teilhard de Chardin, toma prestada, para su título, esta frase del libro más importante de este autor, El fenómeno humano, donde éste explica su concepto del "punto omega", que es aquel donde la conciencia humana ha evolucionado de manera irreversible hasta un punto trascendente donde se funde con la conciencia divina: "Remain true to yourselves, but move ever upward toward greater consciousness and greater love. At the summit you will find yourselves united with all those who, from every direction, have made the same ascent. For everything that rises must converge" (cit. en Early: 111).

Imposible entender cabalmente el título del libro y del cuento con el mismo nombre si no se tiene en cuenta esta referencia, y aun así puede resultar difícil comprenderlos desde esta compleja perspectiva. "Revelation", uno de sus mejores relatos, puede considerarse el ejemplo más claro en este libro del encuentro de la protagonista con su momento de gracia y con el punto omega. El cuento empieza con la llegada de los Turpin al consultorio médico y la entrada de la señora Turpin: "The doctor's waiting room, which was very small, was almost full when the Turpins entered and Mrs. Turpin, who was very large, made it look even smaller by her presence" (The Complete: 488). A partir de esta primera oración, la descripción de la protagonista, como en el caso de muchos de los personajes de éste y otros cuentos, se acerca al estereotipo sureño y es sutilmente exagerada y grotesca (uno de los rasgos característicos del gótico sureño y de los personajes en la narrativa de O'Connor). Como sólo hay una silla disponible, manda a Claud, su marido, que tiene una pierna lastimada por la patada de una vaca, 
a sentarse ahí: "Claud was florid and bald and sturdy, somewhat shorter than Mrs. Turpin, but he sat down as if he were accustomed to doing what she told him to" (489).

La económica descripción de los pacientes en el consultorio aparece, un poco a la manera de Chaucer en The Canterbury Tales, como un cuadro costumbrista de los diferentes representantes de la reducida sociedad rural: "a lean stringy old fellow with a rusty hand spread out on each knee", "a well-dressed gray haired lady whose eyes met hers", "a nurse with the highest stack of yellow hair Mrs. Turpin had ever seen", "a thin leathery old woman in a cotton print dress. She and Claud had three sacks of chicken feed in their pump house that was in the same print", "a lank-faced woman [wearing] a yellow sweat shirt and wine-colored slacks, both gritty looking... Her dirty yellow head was tied behind with a little piece of red paper ribbon. Worse than niggers every day, Mrs Turpinthought..." (490). Y, por supuesto, la adolescente gorda y fea que lee un libro, Human Development: "The poor girl's face was blue with acne and Mrs. Turpin thought how pitiful it was to have a face like that at that age..." (490), además de la descripción de los zapatos correspondientes a cada personaje.

Es muy importante en el cuento la presencia de estos tipos sociales ya que la señora Turpin suele compararse con la gente de su comunidad, agradeciéndole a Dios haberla hecho quien es y no una negra, o lo que sería aún peor, una "white trash": "Sometimes Mrs. Turpin occupied herself at night naming the classes of people. On the bottom of the heap were most colored people [...]; then next to them - not above, just away from - were the white trash; then above them were the home owners, and above them the home and land owners, to which she and Claud belonged..." (491).

En el consultorio las mujeres hablan del problema de que los negros ya no quieran recoger algodón. Es muy importante, en éste y otros cuentos de O'Connor, el uso de la ironía cuando se refiere a la relación de los blancos con los negros, a su racismo prácticamente inconsciente. La señora Turpin también les dice que como ya no pueden contar con los negros, ella y su marido se conforman con tener un poco de cada cosa, particularmente en relación con los animales de granja, especialmente los cerdos, que no son sucios y apestosos como dice la mujer white trash: "One thang I know. Two thangs I ain't going to do: love no niggers or scoot down no hog with no hose" (494). En esas están cuando llega al consultorio un chico negro ("a grotesque revolving shadow") y la white trash comenta que habría que mandar a todos los negros de regreso a África, a lo que la señora Turpin le contesta que "There are a heap of things worse than a nigger" (refiréndose, claro, a la propia mujer white trash).

La señora Turpin no es una mala mujer, es una persona "normal": sureña, blanca, de clase media. Es fuerte y decidida y afectuosa... "But no other O'Connor heroine is more repulsively guilty of pride, more obsessed with status and property" (Di Renzo: 208). A lo largo de la conversación en el consultorio, la chica fea (que se llama, no tan casualmente, Mary Grace) está cada vez más molesta con los comentarios de todos, pero su enojo parece concentrarse en la señora Turpin, quien no alcanza a entender la razón del malestar y el desprecio de ésta. En un momento dado, cuando la conversación gira en torno a ella y su falta de agradecimiento por lo que Dios le ha dado, le arroja 
el libro a la señora Turpin y se le echa encima, tratando de ahorcarla. Cuando los demás logran separarla:

Mrs. Turpin's head cleared and her power of motion returned. She leaned forward until she was looking directly into the fierce brilliant eyes. There was no doubt in her mind that the girl did know her, knew her in some intense and personal way, beyond time and place and condition. "What you got to say to me?" she asked hoarsely and held her breath, waiting, as for a revelation.

The girl raised her head, her gaze locked with Mrs. Turpin's. "Go back to hell where you came from, you old wart hog", she whispered (500).

Esta terrible "revelación" va a sacudir a la señora, quien hasta ese momento se consideraba feliz y agradecida con su Dios que la había hecho como era (y no negra ni white trash). Ya en su casa, descansando en la cama, "the image of a razor-backed hog with warts on its face and horns coming out behind its ears snorted into her head" (502). Pero no puede entender el porqué de semejante insulto: "She had been singled out for the message... The message had been given to Ruby Turpin, a respectable, hard-working, church-going woman. The tears dried. Her eyes began to burn instead with wrath" (502).

Así, llena de rabia, más tarde decide regar a los cerdos con la manguera y, sola, le reclama a Dios haberle enviado semejante mensaje, justamente a ella, una buena mujer. "A final surge of fury shook her and she roared. 'Who do you think you are?' The color of everything, field and crimson sky, burned for a moment with a transparent intensity. The question carried over the pasture and across the highway and the cotton field and returned to her clearly like an answer from beyond the wood" (507).

Entonces, observando con detenimiento a los cerdos, "as if through the very heart of mystery", tiene una visión reveladora.

Podemos recordar aquí la frase de Teilhard de Chardin que da título al libro: "Everything that rises must converge", pues casi al final de este cuento la señora Turpin, después de haber pasado un día de perros, o mejor dicho, de cerdos, tiene la visión de un puente que asciende desde la tierra hacia el cielo y en él una multitud de almas:

There were whole companies of white-trash, clean for the first time in their lives, and bands of black niggers in white robes, and battalions of freaks and lunatics shouting and clapping and leaping like frogs. And bringing up the end of the procession was a tribe of people whom she recognized at once as those who, like herself and Claud, had always had a little of everything and the God-given wit to use it right... They alone were on key. Yet she could see by their shocked and altered faces that even their virtues were being burned away (508).

Se trata sin duda de una descripción con un tono paródico, carnavalesco: "Rather than a sublime revelation of angels and spheres, an awe-inspiring display of celestial hierarchy... Mrs. Turpin sees an All Fools Day celebration..." (Di Renzo: 215). Y este desfile de freaks reúne, de alguna manera, al resto de los personajes que aparecieron antes en el cuento, para mostrar que, al final, todos acaban siendo lo mismo y yendo al 
mismo lugar. (Esto nos recuerda la obra teatral medieval, el "morality play" Everyman, de autor anónimo, en la que el personaje de Everyman se queda perfectamente solo a la hora de su muerte, únicamente con sus buenas obras para acompañarlo hasta el juicio final.) Pero en este caso, el lector no puede estar seguro de que se trate de una verdadera revelación o de un momento de gracia. Tal vez ese momento tuvo lugar en el consultorio médico al recibir el insulto de la chica fea, pero no sabemos si eso cambió efectivamente la vida de la señora Turpin.

No podemos dejar de pensar que el título mismo del cuento, "Revelation", tiene algo de irónico, así como su final: "In the woods around her the invisible cricket choruses had struck up, but what she heard were the voices of the souls climbing upward into the starry field and shouting hallelujah" (509). De acuerdo con Di Renzo: "Mrs. Turpin's vision appears to be a moment of Christian grace, but this moment of grace completely wrecks our conventional understanding of Christianity" (215). Sin embargo, éste es uno de los relatos más optimistas de O'Connor, con todo y final feliz. Si bien es cierto que no podemos estar seguros de que las revelaciones hayan surtido algún efecto trascendental, la señora Turpin regresa a su casa aparentemente más tranquila y reconciliada consigo misma, lo que no quiere decir que sea una persona diferente.

Muchos de sus cuentos tienen, sin embargo, finales terribles. En "The Comforts of Home", la madre de Thomas, un hombre de treinta y cinco años que vive con ella y que en repetidas ocasiones la relaciona con el demonio mismo, trata de ayudar a Star, una joven delincuente, y termina llevándola a su casa que para Thomas era un espacio de aislamiento y seguridad total: "His home was to him home, workshop, church, as personal as the shell of a turtle and as necessary. He could not believe it could be violated in this way" (Everything: 122). Lo curioso es que esta muchacha patética ("the little slut"), la principal freak en el cuento, llega a convertirse a lo largo del relato en una especie de doble del mismo Thomas, algo así como su alter ego femenino, que amenaza no solamente su paz y tranquilidad doméstica sino incluso su propia identidad.

Star se presenta a sí misma como una ninfomaniaca con una historia sexual de violaciones y otras monstruosidades, como una psicópata que no tiene lugar en la sociedad, ni siquiera en el asilo o en la cárcel. Desde su peculiar anormalidad, se convierte para Thomas en un espejo deformante de lo que para él era una existencia ordenada, "normal". Lo peor de todo es que esta mujer, a pesar de representar para él la imagen misma de la corrupción, era de alguna manera inocente: "He needed nothing to tell him he was in the presence of the very stuff of corruption, but blameless corruption because there was no responsible faculty behind it. He was looking at the most unendurable form of innocence" (117). Esta ambigüedad moral que borra la frontera entre el bien y el mal será una constante a lo largo del cuento, donde vemos que no sólo la corrupción tiene su lado de inocencia sino que la virtud también puede acabar siendo culpable. Sin embargo, es la propia madre de Thomas quien no deja de insistir, desde un principio, en que su ayuda desinteresada surge de la "virtuosa" preocupación de que podría tratarse de él, de qué pasaría si a su hijo llegara a sucederle algo semejante. Por ejemplo, cuando Thomas se queja ante su madre de que la chica invadió su cuarto 
desnuda, ella trata de explicar sus motivaciones: "I keep thinking it might be you [...] If it were you, how do you think I'd feel if nobody took you in?" (113). Aparentemente la madre es la verdadera responsable de esta situación caótica, de la ruptura del orden tan valioso para él.

A pesar de que Star es una freak por el hecho mismo de ser una desadaptada, en este cuento no sólo la madre sino el mismo Thomas son personajes que a los ojos del lector resultan bastante extraños. Tal parece que la mirada irónica de Flannery O'Connor pretende que todos sus personajes aparezcan ante nosotros como criaturas frágiles en un mundo sin sentido. En un momento en que Thomas observa y es observado por el rostro de Star leemos: "There was something about the look of it that suggested blindness, but it was the blindness of those who don't know that they cannot see. Thomas was curiously sickened" (118). Esa misma ceguera, obviamente, podría adjudicarse al mismo Thomas así como a muchos de los personajes de esta autora que parecen víctimas de una fatalidad absurda.

Él es un hombre dedicado a estudiar que ha vivido hasta cierto punto aislado del mundo exterior y que se ve envuelto en una situación ineludible provocada por otros; sólo que en este caso, a diferencia de lo que suele suceder en muchas tragedias, no parece haber una explicación trascendente a dicho estado de cosas y las soluciones que se le presentan resultan más bien impuestas por una voluntad ajena a la suya, por lo que su situación se vuelve grotesca. Descubrimos en él una obsesión por la virtud y un rechazo a las mujeres, en especial a todo aquello que se asocia con la sexualidad femenina que parece amenazar su propia integridad moral. En relación con esto vale la pena señalar que, a diferencia del esquema gótico tradicional donde una mujer joven debe confrontar el erotismo masculino, aquí, al contrario, es un hombre joven, huérfano de padre, quien debe enfrentarse al erotismo femenino.

Sin embargo, progresivamente escucha la voz del padre muerto, las órdenes que lo obligan a actuar como guiado por esa fuerza patriarcal que parece haber encarnado en él. En este sentido, el involuntario asesinato de su madre no resulta tan inexplicable ya que, independientemente de la presencia insoportable de Star en su casa, el conflicto profundo de Thomas es sin duda con la madre sobreprotectora de la cual, obviamente, no ha sido capaz de separarse y con quien mantiene un vínculo edípico.

Con mucha ironía la voz narradora en este cuento nos aclara que Thomas sí quería a su madre:

He loved her because it was his nature to do so, but there were times when he could not endure her love for him. There were times when it became nothing but pure idiot mystery and he sensed about him forces, invisible currents entirely out of his control. She proceeded always from the tritest of considerations - it was the nice thing to do - into the most foolhardy engagements with the devil, whom, of course, she never recognized (119).

Sólo que cuando la obliga a escoger entre la muchacha y él, Thomas tiene que reconocer su derrota y, guiado por la voz del padre muerto a quien parece haber ido 
encarnando poco a poco y a pesar suyo, terminará disparando la pistola y matando a su madre por error, restableciéndose así, de manera absurda, el orden roto: "Thomas heard it as a sound that would shatter the laughter of sluts until all shrieks were stilled and nothing was left to disturb the peace of perfect order" (118). No obstante, este momento de "gracia" resulta doblemente irónico no sólo porque Thomas dispara y mata a su madre sino porque hay un cambio de punto de vista en el cuento y el sheriff, que entra a la casa inmediatamente después del disparo, decide que en realidad Thomas y la chica querían deshacerse de la madre: "He was accustomed to enter upon scenes that were not as bad as he had hoped to find them, but this one met his expectations" (119).

Por último, menciono brevemente el final del último cuento de esta colección, "Judgement Day”, que parece ser una reescritura del primer cuento que publicó O'Connor, "The Geranium". Resulta interesante descubrir que en este caso la "revelación" del juicio final tiene lugar en la mente senil y bastante desquiciada de Tanner, quien se cae por la escalera y alucina que está en un tren, dentro de un ataúd, de regreso a su tierra, South Alabama. Excepto que no está muerto y, al abrir la caja sus amigos negros, éste los sorprende brincando como un muñeco Jack in the Box y gritando: "Judgement Day! Judgement Day! You idiots didn't know it was Judgement Day, did you?" (The Complete: 549) antes de volver en sí y darse cuenta de que a quien está viendo es a su vecino negro, al que le dice Preacher y quien, podemos deducir, acabará matándolo.

Los momentos de gracia en los cuentos de O'Connor no son evidentes y depende del lector reconocer si los personajes efectivamente tuvieron esa revelación sobre sí mismos que trae consigo una transformación interior, algunos para seguir viviendo con la aceptación de sus limitaciones y de su incompletud, y otros inmediatamente antes de su muerte. Para que esto suceda tienen que haber vivido momentos decisivos que trastornan su existencia y haber sufrido alguna forma de violencia que puede llegar a aniquilarlos física o psicológicamente pues, según la autora, es lo único capaz de acercarlos a la realidad y quitarles el velo de los ojos: "In my own stories I have found that violence is strangely capable or returning my characters to reality and preparing them to accept their moment of grace. Their heads are so hard that nothing else will do the work" (Mystery: 44).

Para O'Connor, el hombre moderno busca desesperadamente al dios perdido y un conocimiento al que no puede tener acceso como no sea a partir de experiencias perturbadoras y violentas que les revelen el gran misterio de la gracia divina: "The novelist with Christian concerns will find in modern life distortions which are repugnant to him, and his problem will be to make these appear as distortions to an audience which used to see them as natural; and he may well be forced to take ever more violent means to get his vision across this hostile audience" (Mystery: 33).

Lo interesante es que, incluso para los lectores que no tienen presente esta dimensión religiosa de la narrativa de O’Connor, sí es posible identificar ese desenlace, aunque sólo sea como un momento climático en el desarrollo del relato y que es muy parecido, como decía antes, a las "epifanías" de Joyce o los "moments of being" de 
Woolf. Se trata entonces de una manera de narrar, de una estructura que logra, al final de cada cuento, sorprendernos y a veces también asustarnos.

\section{Obras citadas}

BAJTIn, Mijail. La cultura popular en la Edad Media y Renacimiento. Barcelona: Barral Editores, 1974. Impreso.

Chase Coale, Samuel. In Hawthorne's Shadow. Kentucky: The University Press of Kentucky, 1985. Impreso.

Di Renzo, Anthony. American Gargoyles: Flannery O'Connor and the Medieval Grotesque. Illinois: Southern Illinois University Press, 1995. Impreso.

EARly WhitT, Margaret. Understanding Flannery O'Connor. South Carolina: University of South Carolina Press, 1995. Impreso.

Kahane, Claire. "The Gothic Mirror", The (M)other Tongue. Eds. Shirley NeLson, Claire Kahane y Madelon SPREnGnether. Ithaca: Cornell University Press, 1986. Impreso.

O’Connor, Flannery. The Complete Stories. Nueva York: The Noonday Press, 1991. Impreso.

. Mystery and Manners: Occasional Prose. Eds. Sally y Robert FITZGERALD. Nueva York: Farrar, Straus and Giroux, 1970. Impreso.

. Everything That Rises Must Converge. Nueva York: Signet, 1967. Impreso.

RubIN, Louis D. Jr. The American South: Portrait of a Culture. Nueva Orleans: Forum Series, 1979. Impreso. 
